\title{
Commentary
}

\section{Color Vision Impairment in Parkinson's Disease}

\author{
Anna Piro ${ }^{\mathrm{a}, *}$, Antonio Tagarellia ${ }^{\mathrm{a}}$, Giuseppe Nicoletti ${ }^{\mathrm{a}}$, Robert Fletcher ${ }^{\mathrm{b}}$ and Aldo Quattrone ${ }^{\mathrm{a}}$ \\ ${ }^{a}$ Neuroimaging Research Unit, IBFM-CNR, Germaneto, Catanzaro, Italy \\ ${ }^{\mathrm{b}}$ City University, The Northampton Institute, London, UK
}

Acquired disturbances of color vision are a highly varied group of defects with frequent departures from established patterns. They can progress from normal trichromatism to anomalous trichromatism on to a dichromatic stage and to monochromatism where most color vision is lost, or they may be relatively stable [1]. A significant "modern effort" to present such conditions was published for first time in 1972 [2], attention was drawn in a significant account given 15 years later by Jaeger and Krastel emphasizing pharmaco-therapeutical effects [3]. Normally, dopaminergic neurons act in the outer and inner retina at multiple levels, producing alterations to the flow of visual information in a complex fashion. Dopamine is a chemical messenger for light adaptation, promoting the flow of information through cone circuits while diminishing that through rod circuits [4]. Color vision relies on the cone photoreceptor population and is therefore largely confined to the central retina. Because there is a segregation of color-specific information at the retinal into blue-yellow and red-green pathways, it is possible to use color discrimination tasks to assess cone and retinal ganglion cell subpopulations [4]. It has been reported that color vision is impaired in Parkinson's disease (PD) [5] and some works have suggested that these disturbances to color vision affect predominantly the short-wave cone pathway [6]. Birch suggested that the acquired type three (tritan) defect at the retinal level as an early diagnostic sign of Parkinson's

\footnotetext{
${ }^{*}$ Correspondence to: Anna Piro, Neuroimaging Research Unit, IBFM-CNR, Germaneto, Catanzaro, Italy. Tel.: +393402529194; Fax: +39 0 9613695919; E-mail: anna.piro@cnr.it.
}

disease [7]. We examined 49 PD Calabrian all male patients (age range, $50-85$ years, mean age, 67 years) to avoid that genetic appearance of the "lionization" which can be present in the heterozygous females for color vision deficiency who mimic the vision of the normal females [8]. All patients underwent the L-DOPA doses subdivided into two different groups: $125-300 \mathrm{mg} /$ day, low daily dose; $400-1000 \mathrm{mg} /$ day, high daily dose. 25 age and sex matched controls showing a normal color vision, in comparison with the patients (chi square $=25.3, \mathrm{df}=1, p<0.0005$ ) were enrolled in comparison with 25/49 PD patients showing a defect of bleu/yellow axis. Controls did not show any PD signs, or other acquired ophthalmological disease. After informed consent, all patients, and controls had an examination by an ophthalmologist in order to rule a diabetic retinopathy, or a cataract, or an optic neuritis, or a senile maculopathy, or ocular fundus' anomalies that could influence the color vision analysis. All PD patients who made more than 5 mistakes during their reading from the first 17 Ishihara plates were diagnosed as being color-blind, and this diagnosis was confirmed by their reading from the next four (numbers 18-21) Ishihara plates. The last four Ishihara plates (numbers 22-25) were utilized to define the type of color-blindness (the red or green colorbindness) and the grade of color-blindness: protanopy, the absence of red vision or protanomaly, the small deficiency of red color vision, and deuteranopy, the absence of green color vision or deuteranomaly, the small deficiency of green color vision [9]. PD colorblind patients were excluded from the analysis. So, 
our sample constituted by 49 PD male patients had normal color vision prior to PD. Dysfunction of the visual system in PD concerning achromatic as well as abnormal chromatic vision has been demonstrated by clinical, psychophysiological and neurophysiological methods [6]. 25/49 PD patients (51\%) showed a defect of bleu/yellow axis reading the respective Farnsworth's dichotomous test, 15D [10], and City University test [11] plates; Ishihara [9] test confirmed their not colorblind status. 19/25 PD patients underwent a L-DOPA mean dose of $700 \mathrm{mg}$ /day (range 400-1000 mg/day), showing a PD mean duration of 8.4 years (range $0.5-18$ years), and a mean treatment duration of 7.1 years (range $0.25-17$ years); PD mean onset, 60 years (range 39-76 years). Reading the tests plates they show: Ishihara test $=1.8$ mean mistakes (range 0-6); Farnsworth's dichotomous test, $15 \mathrm{D}=7.9$ mean mistakes (range 5-14); City University test $=3.9$ (range 1-11). 6/49 PD patients undergoing a L-DOPA mean dose of $237,5 \mathrm{mg}$ /day (range $125-300 \mathrm{mg} /$ day): Ishihara test $=1.2$ mean mistakes (range 0-2); Farnsworth's dichotomous test, $15 \mathrm{D}=7.1$ mean mistakes (range 1-10); City University test $=3.1$ (range 1-6); PD mean duration of 8.3 years (range $4-13$ years); mean treatment duration of 4.6 years (range 1.5-10 years); PD mean onset, 58,5 years (46-67 years). The different L-DOPA dose did not influence color vision in PD patient, too. 3/49 PD patients $(6,1 \%)$ showed a defect of red/green axis reading the respective Farnsworth's dichotomous test, 15D, and City University test plates: 7.3 mistakes (range 6-9), and 1.6 (range 1-2), respectively. These subject showed a PD mean duration of 7.3 years (range 4-13); a mean treatment duration of 5.0 years (range 1-12 years), and a L-DOPA mean dose of $875 \mathrm{mg} /$ day (range 125-2000 mg/day); PD mean onset, 59,6 years (range 43-70 years). These subjects were grouped because their low sample size. 4/49 patients were colorblind, 9/49 were excluded because showed acquired ophthalmologic disease (cataracts, or other); (8/49) of patients showed normal color vision.

According with Haug [6] who suggested that the influence of PD is most noticeable in signals by the short wave cone pathway because the short wave cones are widely separated. In the retina the small bistratified ganglion cells, which are the morphological substrate of the short wave cone pathway [12] have much larger receptive fields than the midget ganglion cells, and may be more dependent upon long range spatial interactions mediated dopaminergic interplexiform or amacrine cells. $3 / 49$ patients showing a defect of red/green axis making a protan error confirming
Silva's [13] data probing chromatic and achromatic contrast sensitivity changes in Parkinson's disease using complex psychophysical measures designed to isolate parvocellular, koniocellular and magnocellular pathways. Significant impairment in all three pathways was found, more marked along the protan/deutan axis than the tritan. This pattern contrasts with that typically seen in aging, predominant tritan axis deficiency, or in retinal disease states such as glaucoma in which all color axes are involved with particular emphasis on the tritan axis or best macular dystrophy where color axis involvement depends on the stage of the disease itself $[13,14]$. Such comparisons suggest a disease specific pattern of retinal impairment in Parkinson's disease distinct from normal ageing or the commoner age related ophthalmological disease.

According with the literature [6], we conclude that color vision is an indicator of the pathophysiology of PD revealing to be a true multisystem disorder with a wide variety of motor and not motor features.

\section{CONFLICTS OF INTEREST}

The authors have no conflict of interest to report.

\section{REFERENCES}

[1] Fletcher R, \& Voke J (1985) Defective color vision, fundamentals, diagnosis and management. A Hilger, Bristol, United Kingdom.

[2] Smith VC, \& Pokorny J (1972) Spectral sensitivity of colorblind observers and the cone photopigments. Vision Res, 12, 2059-2071.

[3] Jaeger W, \& Krastel H (1986) Color vision deficiencies caused by pharmacotherapy. In Color Vision Deficiencies, Verriest A, ed. Junk and Nijhoff, Dordrecht, The Netherlands, pp. 37-52.

[4] Archibald NK, Clarke MP, Mosimann UP, \& Burn DJ (2009) The retina in Parkinson's disease. Brain, 132, 1128-1145.

[5] Büttner T, Patzold T, Kuhn W, Muller T, \& Przuntek H (1994) Impaired color discrimination in Parkinson's disease. Neuroophthal, 14, 91-95.

[6] Haug BA, Kolle RU, Trenkwalder C, Oertel WH, \& Paulus W (1995) Predominant affection of the blue cone pathway in Parkinson's disease. Brain, 118, 771-778.

[7] Birch J, Kolle RU, Kunkel M, Paulus W, \& Upadhyay P (1998) Acquired color deficiency in patients with Parkinson's disease. Vision Res 38, 3421-3426.

[8] Lyon MF (1961) Gene action in the X-chromosome of the Mouse (Mus Musculus L). Nature, 190, 372-373.

[9] Ishihara S (1990) Ishihara's test for colorblindness. $38^{\circ}$ Plates Edition. Kanehara and Co., Tokyo, Japan.

[10] Farnsworth D (1943) The Farnsworth-Munsell 100 Hue and dichotomous test for color vision. J Opt Soc Am, 33, 568578.

[11] Fletcher RJ (1975) The City University Color Vision test. Keeler Instruments, London, United Kingdom. 
[12] Dacey DM, \& Lee BB (1994) The "blue-on" opponent pathway in primate retina originates from a distinct bistratified ganglion cell type. Nature, 367, 731-735.

[13] Silva MF, Faria P, Regateiro FS, Forjaz V, Januario C, Freire A, \& Castelo-Branco M (2005) Independent patterns of damage within magno, parvo and koniocellular pathways in Parkinson's disease. Brain, 128, 2260-2271.
[14] Castelo-Branco M, Faria P, Forjaz V, Kosac LR, \& Azevedo $H$ (2004) Simultaneous comparison of relative damage to chromatic pathways in ocular hypertension and glaucoma: Correlation with clinical measures. Invest Ophthalmol Vis Sci, 45, 499-505. 